\title{
Lightning-induced transient effects in a hybrid PV-wind system and mitigation strategies
}

\begin{abstract}
Lightning transient effects on a hybrid 4.1 MW PV-wind system were investigated in this work by using PSCAD/EMTDC software. A simulation was performed with real lightning current waveforms, namely, negative first stroke and positive stroke, with and without a lightning protection system. Surge protective devices were also designed based on European Commission for Electrotechnical Standardization (CENELEC) standards. Analyses were performed to determine appropriate ratings for the threat level and specifications of the equipment/components of the hybrid system that can suppress lightning transients to an acceptable voltage level.
\end{abstract}

Keyword: Hybrid PV-wind system; Transient voltage; Transient current; Lightning effect; Heidler function; Lightning protection system 